\title{
Point-to-source path tracing Monte Carlo to compute the Clausing and distribution functions in high-vacuum systems
}

\author{
Maxime Rondeau, L. Isnard, and R. Arès* \\ Laboratoire d'Épitaxie Avancée, Institut Interdisciplinaire d'Innovation Technologique (3IT), \\ Université de Sherbrooke, 3000 Boul. de l'Université, Sherbrooke, Québec, Canada, J1K 0A5
}

\begin{abstract}
This paper presents an approach to simulate the free molecular flow in vacuum systems by using a Monte Carlo method for solving the Boltzmann particle transport equation with no intermolecular collisions. Sometimes referred to as a point-to-source Monte Carlo path tracing in image rendering, in this paper the name is borrowed from the thermal radiation and heat transfer field, reverse Monte Carlo path tracing. It is shown that this method provides better accuracy and stability when computing the Clausing function when compared to the standard test particle Monte Carlo method used for free molecular flow. The Clausing function leads to the distribution function of positions and velocities from which the particle density map, pressure gradient, energy flux, and other local quantities can be computed. Using reverse path tracing, the particle concentration in a conical segment is computed, and the maximal flow input is determined by calculating the mean free path at the maximum density position.
\end{abstract}

\section{INTRODUCTION}

Many applications, such as spectroscopy, microscopy, epitaxy, lithography, distillation, arc remelting, and particle accelerators, benefit from or require high vacuum when operating. In those environments knowledge of the particle distribution anywhere on the walls may, for example, help predict coating areas due to gases being injected or particles etching off surfaces during processes, optimize the location of pumps to better maintain vacuum, and therefore improve the overall design of systems. Simulating the particle distribution in the whole or key sections of such systems requires the solution to the collisionless Boltzmann transport equation. However, solving this equation is a computationally expensive task in environments with complex geometries. This is why efficient solving methods are interesting when it comes to optimizing the design of vacuum systems.

Such systems operate in a regime called free molecular flow which was first clearly demonstrated by Fleming [1] when he observed a shadowing effect made by a horseshoe carbon wire blocking the evaporation of a thin copper film in a light bulb. Such a phenomenon can be explained when the mean free path $(\lambda)$ of molecules is much higher than the characteristic dimensions of the system $(L)$, meaning the molecules radiate from the copper layer and directly hit the bulb. The Knudsen number $\left(K_{n}\right)$, the ratio between $\lambda$ and $L$, is thus very large $\left(K_{n} \gg 1\right)$ for highly rarefied gases. A more mathematical description of this flow regime was introduced by Knudsen [2], who studied the flow of gases through narrow tubes and estimated the energy throughput to be proportional to the pressure gradient, with a proportionality coefficient being purely geometrical. Only the shape introduces flow resistance as expected for a regime with a large $K_{n}$. However, it was Clausing [3] who developed an integral equation, with a line-of-sight approach, to compute flow resistance induced by a cylinder of length $L$ and radius $R$. Solving the integral equation actually gives access to the Clausing function, the probability $w(x)$ for a molecule that leaves any position $x$ along the length of a tube to exit at

*Richard.Ares@usherbrooke.ca 
the end:

$$
w(x)=w_{r s}(L-x)+\int_{0}^{L} d \xi w(\xi) w_{r r}(\xi-x),
$$

where $w_{r s}(L-x)$ is the probability for a molecule to directly leave a ring section of the tube at position $x$ and exit at position $L$ through a disk. On the other hand $w_{r r}(\xi-x)$ is the probability for a molecule to go directly from a small ring section at position $x$ to another one at position $\xi$. Solving Eq. (1) therefore gives access to $w(x)$, which helps to compute the Clausing factor (W), defined as the probability for a molecule that diffusely enters the tube to exit at the other end, which is finally used to compute the conductance of pipes. The flow conductance helps to estimate pumping times for vessels using pipes of various aspect ratios. The Clausing function $w(x)$ also gives access to the distribution function and therefore to the local density, mean free path, mass transport, and other quantities that help characterizing vacuum systems.

Using local particle conservation, one can derive the transport equation for free molecular flow, which can be seen as a generalized Clausing equation (not only for pipes). Solving this equation is no easy task. Simple geometries, such as shapes with axial symmetry, reduces the Clausing equation to a one-dimensional integral, allowing numerical integration to be carried out with the use of orthogonal polynomials. This effectively transforms the integral into a matrix equation. Such a method was used by Iczkowski [4] for the effusion through conical orifices. When no symmetry can be exploited it is usually necessary to use numerical methods to carry out the integrals. Combining the numerical integral with an iteration process and a cutoff procedure, the solution to the Clausing equation was computed by various authors for applications that use gas injection or evaporation, molecular beam epitaxy by Curless [5], chemical beam epitaxy by Isnard [6], and physical vapor deposition in small trenches by Cale [7]. This last computing method is very fast, but is harder to implement when the number of objects in the system increases. Another approach is to solve the equation with Markov chains to sample the integral. Again, with surface discretization, this Monte Carlo approach was used by Lobo and Gomez-Goni [8] for pipes and conical shapes. Essentially, most of the solutions require defining small areas or patches that make up the objects, a tedious task when faced with complex systems, and also a source of small error. Moreover, in this last Monte Carlo method, smaller area elements scale with greater computing time.

The Clausing equation can be linked to the particle flux $\mathbf{g}$, defined as the number of impinging or emitted particles per unit time per unit area, on any surface in the system, and is in fact nothing more than the collisionless Boltzmann integral transport equation with diffuse surfaces, giving the so-called radiosity equation $\mathbf{g}=\mathbf{g}^{0}+\mathbf{T g}$. In this equation $\mathbf{g}^{0}$ is the distribution of particles on the surfaces of the system with no surface collision prior and $\mathbf{T}$ is the transport operator of the system. In the case of free molecular flow the transport operator is simply a matrix of geometric factors. Since the flow is described by a standard particle transport equation, many solving methods are known for heat, light, and neutron transport. One of the methods to solve the transport equation is to use the adjoint solution from the adjoint transport equation $[9,10]$. However, in the case of free molecular flow, the transport equation is self-adjoint, and the adjoint solution should be the solution to the transport equation.

This work is inspired from a Monte Carlo path-tracing sampling method used in optics [11], neutron diffusion [10], and radiative heat transfer [12], which is applied to the free molecular flow, similarly done by Malhotra [13]. The procedure proposes to begin the particle path at the point where one wants to compute the impinging flux and keep emitting from this location in a diffuse way until one hits an exit or a particle source area. This procedure is called reverse path tracing from now on, a name borrowed from the field of thermal radiation heat transport [12]. This is in contrast with the method used by Gomez-Goni [8] mentioned previously. In this Monte Carlo approach, molecules originate at the particle source and are emitted diffusely until they exit or return to the source. To compute the impinging flux one needs to count the number of collisions made in a small area of length $\varepsilon$ centered around the desired location. Both processes are illustrated in Fig. 1. This latter 


\section{Direct path tracing}

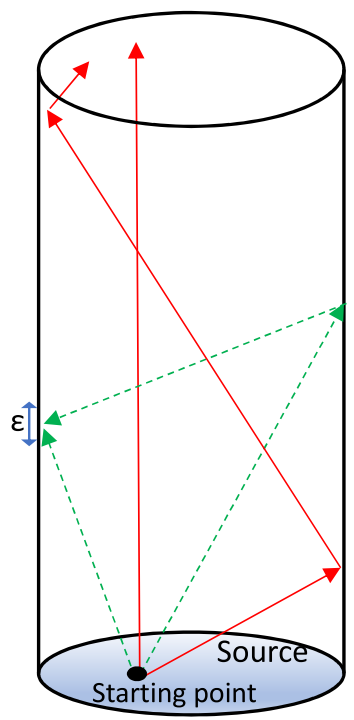

Reverse path tracing

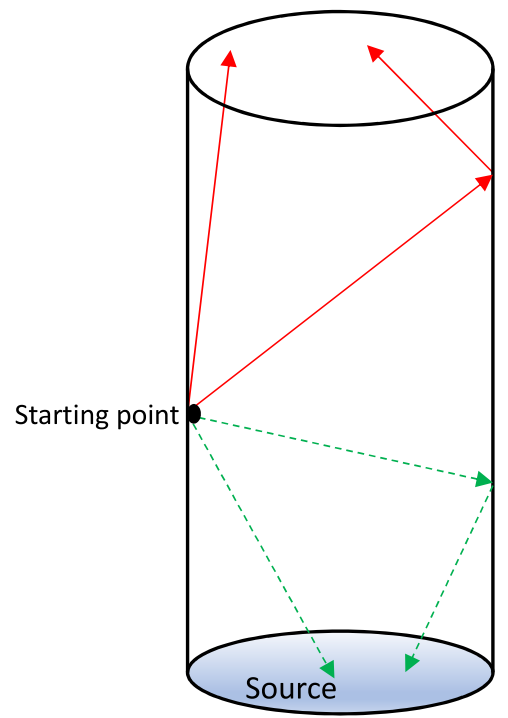

FIG. 1. Illustration of the processes involved in the two path-tracing methods. The particle source is a uniformly distributed diffuse emitter represented by the bottom cap of the cylinder, the top cap surface is called the exit area, and the body of the cylinder is diffuse re-emitting surfaces called walls. Particles are generated at the black spot bounce of the walls, and their trajectories are illustrated in two colors. Line paths do not contribute to the impinging flux whereas dashed paths do. In the direct path example, the particles are captured in a small collecting area of length $\varepsilon$.

method will be called forward or direct path tracing from now on, but is also known as a test particle Monte Carlo method [14,15].

The objective is to demonstrate that when reverse path tracing is used properly it offers greater accuracy and immensely more precision to calculate any quantity when compared to direct path tracing for a given computation time. This is especially true when the wanted solution is only for a subset of points in a large system. Furthermore, being a path tracer, it offers the same statistical information on any desired quantity that direct path tracing would. For example, it is possible to compute the transmission probability as in direct path tracing; see Table I.

Moreover, it does not require the use of small areas to collect particles while maintaining the advantage of using Boolean operations between simple geometrical shapes, such as cones, pipes, planes, and spheres, to construct complex systems. However, it does suffer from the same shortcoming of any Monte Carlo method, slow convergence (when compared to other numerical integration methods) proportional to the inverse square root of the number of generated paths.

TABLE I. Clausing factors $(W)$ expressing the probability for a molecule that enters a cylinder of aspect ratio length over radius $L / R$ to exit at the other end. The integer $N$ represents the number of paths generated to obtain the transmission probability in this work, which is compared to Ref. [16].

\begin{tabular}{llcl}
\hline \hline$L / R$ & $N$ & $W$ (this work) & \multicolumn{1}{c}{$W([16])$} \\
\hline 0.1 & $10^{6}$ & 0.9524 & 0.952398907315 \\
1000 & $10^{6}$ & 0.002648 & 0.00264761 \\
\hline
\end{tabular}




\section{THEORY AND DEFINITIONS}

\section{A. The transport equation applied to highly rarefied gases}

A free molecular flow system in a steady state, with isothermal walls and sources, is composed of three surface types:

(1) Particle source areas, or inlets, are characterized by a cosine law of emission at each point of the surface, with an emission rate that may change from point to point. However, the angular distribution of emission rates on the source area remains constant. This means that incoming particles from other surfaces hitting the source area are recycled so that the emission pattern stays unchanged.

(2) Walls re-emit all incident particles with a cosine law assuming there is no sorption and particle migration on such areas. Even though the cosine law of emission does not apply $[17,18]$ for all systems, this paper treats particles that are thermally accommodated by the system walls. A previous paper has shown that such conditions are experimentally attainable [6].

(3) Particle exit areas, or outlets, do not reemit anything, particles hitting such a surface are lost outside the system.

Let $g(\mathbf{r})$ be the impinging or emitted flux of particles at point $\mathbf{r}$ in a system with a steady flow. Then it is possible to compute the flux $g(\mathbf{r})$ :

$$
g(\mathbf{r})=v \int_{S_{i}} d S_{i} p\left(\mathbf{r}_{i} \rightarrow \mathbf{r}\right)+\int_{S^{\prime}} g\left(\mathbf{r}^{\prime}\right) d S^{\prime} p\left(\mathbf{r}^{\prime} \rightarrow \mathbf{r}\right),
$$

where the integral on $S^{\prime}$ spans over all the walls, and $v$ is a uniform (uniformity is not an essential criteria) and constant source of flux, $S_{i}$. The term $\left[g\left(\mathbf{r}^{\prime}\right) d S^{\prime}\right] p\left(\mathbf{r}^{\prime} \rightarrow \mathbf{r}\right) d S$ can be read as follows: the number of particles transferred to an area $\mathrm{d} S$ located at $\mathbf{r}$ that originated from an area element $d S^{\prime}$ around position $\mathbf{r}^{\prime}$ with an emitted flux of $g\left(\mathbf{r}^{\prime}\right)$. Since $g\left(\mathbf{r}^{\prime}\right) d S^{\prime}$ is an emission rate, the number of emitted particles per second, then $p\left(\mathbf{r}^{\prime} \rightarrow \mathbf{r}\right) \mathrm{d} S$ must be the fraction exchanged between the two areas, with Eq. (3) giving the exchange fraction per unit area. All area elements are scalar quantities, the vectorial components of the surfaces being incorporated,

$$
p(a \rightarrow b)=\frac{\left(\mathbf{r}_{b}-\mathbf{r}_{a}\right) \cdot \mathbf{n}_{a}\left(\mathbf{r}_{b}-\mathbf{r}_{a}\right) \cdot \mathbf{n}_{b}}{\pi\left|\mathbf{r}_{b}-\mathbf{r}_{a}\right|^{4}},
$$

where $\left(\mathbf{r}_{b}-\mathbf{r}_{a}\right)$ is the distance vector between the two positions, and $\mathbf{n}_{b}, \mathbf{n}_{a}$ are unit vectors normal to the area elements, always oriented so that the scalar product with the distance vector is positive. Just by looking at Eq. (3), one can convince oneself that the exchange fraction per unit area is reversible:

$$
p(a \rightarrow b)=p(b \rightarrow a) .
$$

Equation (4) also works for specular reflection and may hold for other scattering laws. Therefore, reverse path tracing in free molecular flow is valid for gas-surface interaction models that respect Eq. (4). This paper concentrates on diffuse scattering expressed by Eq. (3), and therefore the particle transport Eq. (2) can be related to the Clausing equation [3]. Rewriting Eq. (2) using a matrix-vector notation,

$$
\mathbf{g}=\mathbf{g}^{0}+\mathbf{T g}
$$

where $\mathbf{g}^{0}$ is the first integral on the right-hand side of Eq. (2) and $\mathbf{T g}$ is the second one, then the solution is (see Refs. $[10,19,20]$ for more details)

$$
\mathbf{g}=(\mathbf{1}-\mathbf{T})^{-1} \mathbf{g}^{0} .
$$

Since the fraction of particles that are transferred between any two small sections of emitting or reemitting walls is always inferior to one, given by the integral operator $\mathbf{T}$ when all possible section pairs are considered, Eq. (6) can be developed into a sum using the Neumann expansion [20]:

$$
\mathbf{g}=\mathbf{g}^{0}+\mathbf{T g}^{0}+\mathbf{T} \mathbf{T} \mathbf{g}^{0}+\mathbf{T} \mathbf{T T} \mathbf{g}^{0}+\cdots .
$$




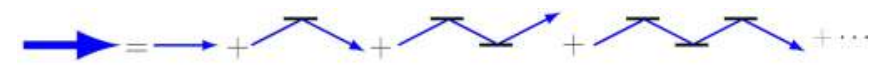

FIG. 2. Schematic of the iterative process expressed by the equation $\mathbf{g}=\mathbf{g}^{0}+\mathbf{T g}^{0}+\mathbf{T} \mathbf{T} \mathbf{g}^{0}+\mathbf{T} \mathbf{T} \mathbf{T} \mathbf{g}^{0}+\cdots$ Each direction change on a wall represents an integral over all the re-emitting walls.

Basically, Eq. (7) means that in a stationary flow, the total impinging flux at a certain position on any surface of the system is a direct transfer of particles with 0 collisions $\left[g^{0}(\mathbf{r})=\int_{S_{i}} d S_{i} v p\left(\mathbf{r}_{i} \rightarrow \mathbf{r}\right)\right]$ plus the transfer with one collision $\left[\mathbf{T} g^{0}(\mathbf{r})=\int_{S^{\prime}} \int_{S_{i}}\left(\nu d S_{i}\right) p\left(\mathbf{r}_{i} \rightarrow \mathbf{r}^{\prime}\right) d S^{\prime} p\left(\mathbf{r}^{\prime} \rightarrow \mathbf{r}\right)\right]$ and so on. Equation (5) can be imaged as shown in Fig. 2, where the thick arrow represents the total flux at one point and the thinner ones represent $\mathbf{g}^{0}$, and each collision on a wall is an integral over the area of the re-emitting walls in the system.

Since the exchange fraction per unit area is reversible, then looking closely at $\mathbf{g}^{0}$ and using Eq. (4) one finds that

$$
\frac{g^{0}(\mathbf{r})}{v}=\int_{S_{i}} d S_{i} p\left(\mathbf{r}_{i} \leftarrow \mathbf{r}\right)
$$

is actually the fraction of particles that are emitted at $\mathbf{r}$ transferred directly on the source area $S_{i}$. The second term in Eq. (7) can also be rewritten,

$$
\frac{\left[\mathbf{T} \mathbf{g}^{0}\right](\mathbf{r})}{v}=\int_{S^{\prime}} \int_{S_{i}} d S_{i} p\left(\mathbf{r}_{i} \leftarrow \mathbf{r}\right) d S^{\prime} p\left(\mathbf{r}^{\prime} \leftarrow \mathbf{r}\right),
$$

which represents the fraction of particles that originate from $\mathbf{r}$ making one collision and then ending up on the source area $S_{i}$. Since the next term of the series is simply the previous one with an additional $\mathbf{T}$ operator applied, it becomes obvious, by recurrence, that each term is the fraction of particles that originates from $\mathbf{r}$ and finishes on the area $S_{i}$ with an increasing number of collisions on the path they follow. The sum in Eq. (7), divided by $v$, is thus simply the total fraction of particles leaving $\mathbf{r}$ that end up on $S_{i}$ where they are not re-emitted. If one generated $N$ random paths starting at point $\mathbf{r}$, then a numerical approximation of Eq. (2) would be the arithmetic average given by

$$
\frac{g(\mathbf{r})}{v}=\lim _{N \rightarrow \infty} \frac{1}{N} \sum_{i=1}^{N} N^{i}
$$

with $N^{i}$ given by

$$
N^{i}= \begin{cases}1, & i \text { th particle exits through source } \\ 0, & i \text { th particle exits elsewhere }\end{cases}
$$

If the emission pattern was not uniform on the inlet surface, then Eq. (11) needs to be modified. Indeed, the path weight would differ from 1 when the particle would exit at the source area. This weight would depend on where on the source area the particle exited in the following manner: it is the ratio between the inlet emission flux at the point of exit, noted $v\left(\mathbf{r}_{\text {Exit }}\right)$, and the average flux emitted by the inlet area $\left(S_{\text {source }}\right), 1 / S_{\text {source }} \int_{\text {Source }} d S v(\mathbf{r})$. This ratio gives 1 in the case of uniform emission at the inlet.

In the case discussed here, every direction generated in a path history is made with a spatial cosine distribution of angles with respect to the surface normal, and this from start to end. Of course, in a path history a collision with an exit or a source area makes the particle vanish. However, if the starting point is not a wall, the cosine distribution remains the one that needs to be generated. Indeed, if the goal is to get the flux on a imaginary surface within the volume of the system, one needs to define an orientation of the surface and generate the paths as if the surface was a wall. However, during the trajectory, the particle cannot hit this imaginary surface since it is not a system wall. Such cases include the need to compute the flux on the exit area or the returning flux on the source 
area. One notes that the flux computed on a imaginary surface with a certain normal unit vector is different if the normal is taken in the opposite direction. The difference between the opposite fluxes gives the net flux going through the imaginary area.

To sum up, in reverse path tracing, when generating paths from the desired point, one needs only to count the number of particles that end up on the source area to compute the impinging flux. This is in contrast to direct path tracing, where the particles are generated at the particle source area and one counts the particles that make it to a small discrete area around the desired point. Since $g(\mathbf{r}) / v$ is the fraction of particles that reach the particle source, then by definition $w(\mathbf{r})=1-g(\mathbf{r}) / \nu$ must be the Clausing function defined in Sec. I.

\section{B. Convergence and error}

Looking closely at Eq. (11), one sees that counting particles that exit through the source area is a binary event, a random walk process. From this point of view, the convergence of the calculation can be estimated from the fluctuations of the random walk process, which is quantified by its standard deviation. Not having the Bernoulli trial probabilities, which are actually what is being computed, one must use the sample variance of Eq. (10) given by

$$
\sigma^{2} \approx \frac{N}{N-1}\left(\frac{1}{N} \sum_{i=1}^{N}\left(N^{i}\right)^{2}-(\bar{N})^{2}\right), \quad \text { with } \bar{N}=\frac{1}{N} \sum_{i=1}^{N} N^{i} .
$$

Convergence of the computation is evaluated using the definition given in the following, called precision from now on and noted $\delta$ :

$$
\delta \equiv \frac{2}{\bar{N}} \sqrt{\frac{\sigma^{2}}{N}} \times 100 .
$$

However, the precision of the computation does not guarantee that the result is accurate. To evaluate accuracy, noted $\Delta$ in Eq. (14), the result is compared to trusted literature values, $g_{L}(\mathbf{r})$, computed with other calculation methods:

$$
\Delta \equiv \frac{\left|g(\mathbf{r})-g_{L}(\mathbf{r})\right|}{g_{L}(\mathbf{r})} \times 100
$$

\section{The distribution function}

The distribution function $f(\mathbf{r}, \mathbf{v})$ is defined as a density of particles located at point $\mathbf{r}$ that have a velocity $\mathbf{v}$ in a given direction. In a free molecular flow regime with uniform temperature, the solution to the transport equation gives the distribution function as

$$
f(\mathbf{r}, \mathbf{v})=\frac{4}{\bar{v}}\left(\frac{m}{2 \pi k_{B} T}\right)^{\frac{3}{2}} \exp \left[-\frac{m v^{2}}{2 k_{B} T}\right] g\left(\mathbf{r}-t \frac{\mathbf{v}}{|\mathbf{v}|} \bigcap \mathbf{S}\right),
$$

where $\bar{v}=\sqrt{8 k_{B} T / \pi m}$ is the average velocity of a particle in a Maxwell-Boltzmann gas, $T$ the temperature, $k_{B}$ the Boltzmann constant, $m$ the particle mass, $\mathbf{S}$ the system surfaces, and $\left(\mathbf{r}-t \frac{\mathbf{v}}{|\mathbf{v}|} \cap \mathbf{S}\right)$ is simply the nearest intersection between the reverse path line of the particle and the surface located at a distance $t$ [21]. From this distribution we shall compute the particle density anywhere in a system's volume,

$$
n(\mathbf{r})=\int f(\mathbf{r}, \mathbf{v}) d \mathbf{v}
$$

and compute the local mean collision rate $\Gamma(\mathbf{r})$ of a particle,

$$
\Gamma(\mathbf{r})=\sigma_{T} \frac{\int d \mathbf{v}_{1} f\left(\mathbf{r}, \mathbf{v}_{1}\right) \int d \mathbf{v}_{2}\left|\mathbf{v}_{1}-\mathbf{v}_{2}\right| f\left(\mathbf{r}, \mathbf{v}_{2}\right)}{n(\mathbf{r})},
$$




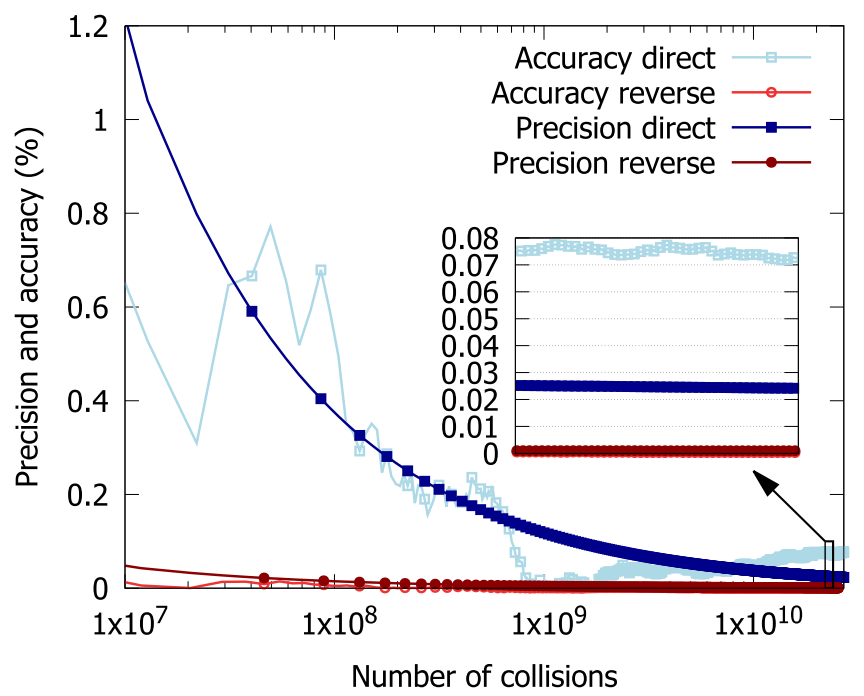

FIG. 3. Accuracy and precision comparison between direct and reverse path tracing of the Clausing function at the entrance point $(z=0)$ of a pipe of aspect ratio $L / R=10$. It is compared to the number of collisions made in the pipe, which is proportional to the computation time. For the direct path tracing, the pipe was separated in 300 equal sectors. Square points represent the direct path simulation, and the circles the reverse path tracing. Empty dots are for accuracy, and the filled ones are for precision.

where $\sigma_{T}=\pi d^{2}$ is the total collision cross section for hard spheres of diameter $d$. The local mean free path is defined with the help of this collision rate:

$$
\lambda(\mathbf{r}) \equiv \frac{\bar{v}}{\Gamma(\mathbf{r})}
$$

\section{REVERSE AND DIRECT PATH-TRACING COMPARISON}

Reverse path-tracing samples only the ones that contribute to the impinging flux at one point, which is not the case in direct path tracing. Because of this, the reverse path will always converge faster than direct path tracing for each single point that is considered. This paper attempts to illustrate this with the comparison of a reverse and direct path Monte Carlo simulation in a pipe of aspect ratio length over radius $L / R=10$.

The simulation was carried out for multiple points along the tube's height (see Fig. 1), identified as the $z$ axis, and the same conclusion was made for each of those points. From this fact, it is sufficient to illustrate the comparison using only one point, in this case the entrance at $z=0$. The reference value of the Clausing function at this point is taken from [8] as $w(0)=0.0850625631$ giving an impinging flux ratio of $g(0) / v=1-w(0)$. For the direct path Monte Carlo the pipe was segmented in 300 sectors of equal width to accumulate the collisions made within the area of each circular band. Such discretization is not needed in reverse path Monte Carlo, only points where the impinging flux is wanted need to be specified. To illustrate the stability and performance of reverse path tracing, accuracy and precision [Eqs. (14) and (13)] are plotted with respect to the number of collisions made within the tube. This represents roughly the number of operations made by the computer to attain a certain degree of accuracy and precision.

Now proceeding with the result of this comparison illustrated in Fig. 3. One striking feature is that reverse path is already stable and converged accurately beneath $0.1 \%$ after $1 \times 10^{7}$ collisions within the tube. On the other hand, the direct path still fluctuates considerably when compared to reverse path tracing and converged to $1.2 \%$ only. This is more than an order of magnitude in computing time 
gain. Moreover, since the direct path uses a set of discrete bands that extend over a small range of the length axis, the impinging flux cannot be evaluated as accurately for a given number of collisions. This is well represented in the inset of Fig. 3 where after $1 \times 10^{10}$ collisions the accuracy is off by $0.075 \%$ but fluctuations are now four times less than this offset. This is due to the numerical error introduced by the finite width of the collecting sector.

Indeed, let $\varepsilon$ be the collecting section length on the $z$ axis. The average number of incident particles on a circular band of area, $\langle g(z)\rangle 2 \pi R \varepsilon$, that is computed with direct path tracing should be

$$
\langle g(z)\rangle 2 \pi R \varepsilon=\lim _{N \rightarrow \infty} \frac{2 \pi R \varepsilon}{N} \sum_{i=0}^{N-1} g\left(z-\frac{\varepsilon}{2}+\frac{i}{N-1} \varepsilon\right) .
$$

With a Taylor expansion to the second order and a few sum identities it is possible to show that the error made on the flux ratio $g(z) / \nu$ is of second order with $\varepsilon$ :

$$
\frac{\langle g(z)\rangle}{v}-\frac{g(z)}{v}=\frac{\varepsilon^{2}}{24} \frac{d^{2}}{d z^{2}}\left[\frac{g(z)}{v}\right]+O\left(\varepsilon^{3}\right) .
$$

As stated in Eq. (20), the numerical error is proportional to the second derivative of the flux along the pipe $\left(d^{2}[g(z) / v] / d z^{2}\right)$. Since the flux along the pipe is not exactly linear as reported by Clausing [3] this derivative is nonzero, therefor producing a small offset. For this reason, while reverse path tracing gives an accurate result for any point along the pipe, the direct path will always average over the collecting patches, giving a small error when the flux is not linear. This error diminishes when the collecting sector width is reduced, but at the price of increasing the convergence time. Since the flux is about linear in a pipe, the numerical error offset is quite small.

\section{CONTROLLED GAS INJECTION IN HIGH-VACUUM SYSTEMS}

With the help of reverse path tracing the flux ratio $g(z) / v$ along the length of a conical section was computed to a precision below $\delta \leqslant 1 \%$ for each of the 500 points chosen. The flux ratio also gives access to the distribution function given by Eq. (15). The starting radius is $0.95 \mathrm{~mm}$, the length is $20.1 \mathrm{~mm}$, and the opening half angle is $10^{\circ}$. This shape was initially studied by Isnard [6] to experimentally show that the transport equation solution is valid for free molecular flow and that the impinging flux arriving on a wafer destined for epitaxial growth could then be controlled by optimizing the shape of a gas injector. However, too high a flux at the entrance illustrated the inadequacy of the free molecular flow hypothesis to describe the exiting flux. To find out what too much flux represents in certain gas injection designs one must compute the distribution function. From this, it is possible to quantify the density anywhere in the conical section and evaluate the mean free path of molecules. An upper limit to the input flux can then be estimated if the injector is to work in free molecular flow.

Figure 4 shows a density map for uniform temperature of the walls and input gas. The cone is illustrated in the inset with the flow direction. The color gradient represents the density ratio within the system and because of the axial symmetry it needs to be plotted only with respect to radius and height. The calculations assume a uniform input flux $v$ emitted at the entrance from a Maxwell-Boltzmann reservoir located upstream. The input flux is then given by $v=n_{i} \bar{v} / 4$, with $n_{i}$ the input density of the gas and $\bar{v}$ the mean velocity of a particle defined after Eq. (15). The density in the cone varies slightly with the radius, being denser in the middle, and very strongly with the height. Indeed, the particle density drops rapidly after a few millimeters of the entrance. Such a drop helps to keep the free molecular flow for most part of the cone. Even at the very beginning, the density drops to $75 \%$ of the input flux. With these data it is possible to estimate the mean free path at the entrance and see when the free molecular flow hypothesis breaks down. Let us attempt to quantify a net flux for which the free molecular flow breaks down, the net flux being what is measured in experiments, $\nu_{\text {net }}=W \nu$, with $W=0.56$ being the cone's Clausing factor defined previously. The source area is given by $A=\pi\left(9.5 \times 10^{-4}\right)^{2} \mathrm{~m}^{2}$ from which the net particle flow can be computed 


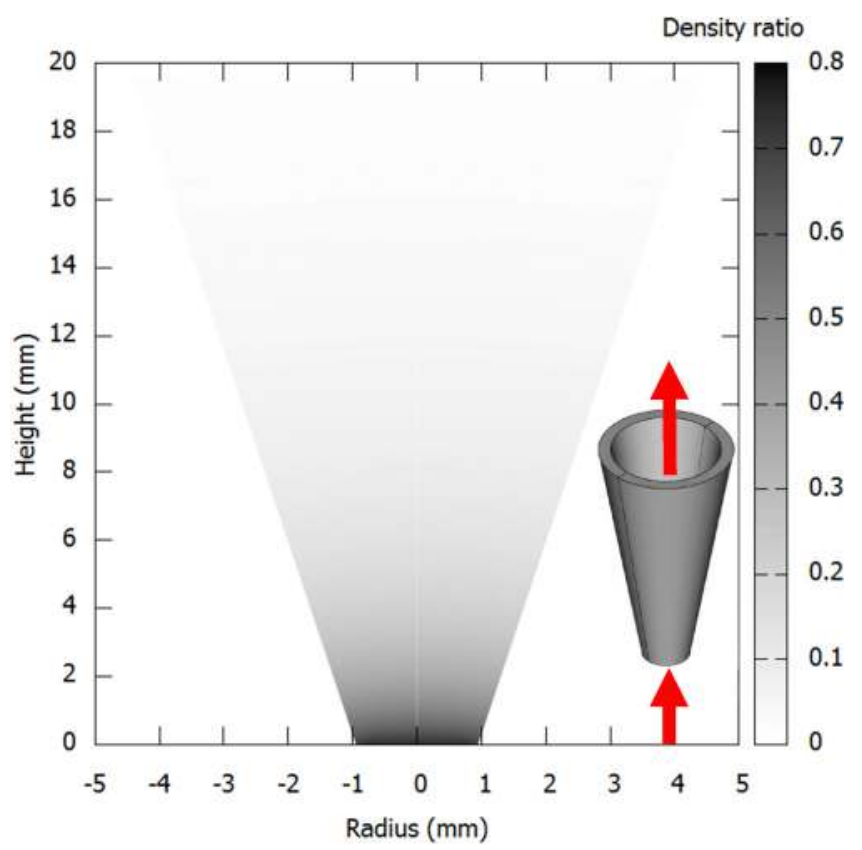

FIG. 4. Relative particle density distribution within a cone. The density at the entrance is 0.75 times input density and decreases rapidly with the height of the frustum. The red arrows in the inset indicate the direction of the flow in the conical section.

with

$$
v_{\text {net }} A=x\left(4.465 \times 10^{17}\right)
$$

where $x$ is the number of net standard cubic centimeter ( $\mathrm{sccm}$ ). Using Eq. (21), the input density may be computed from a Maxwell-Boltzmann gas,

$$
n_{i}=\frac{4 v_{\text {net }}}{\bar{v} W}
$$

with $\bar{v}$ the average velocity of a particle according to Maxwell-Boltzmann distribution and $4.465 \times$ $10^{17}$ the number of particles per second, equivalent to $1 \mathrm{sccm}$. With the definition of the mean free path and Eq. (22), it is now possible to compute the Knudsen number at the entrance,

$$
\frac{\lambda}{L}=\frac{\bar{v}}{\Gamma(\mathbf{r}) L},
$$

considering an input gas of nitrogen $\mathrm{N}_{2}$ [6]. Then the effective intermolecular collision cross section is $\sigma_{T}=4.3 \times 10^{-19} \mathrm{~m}^{2}$ [22,23] and $\bar{v}=475 \mathrm{~m} / \mathrm{s}$ at $295 \mathrm{~K}$. With Eqs. (10), (15), (17), (21), (22), and (23) and using a characteristic length of $L=2 \mathrm{~mm}$ we compute the Knudsen number for various net flows which yields Fig. 5. The plot suggests that for a net flow of about $v_{\text {net }} A=0.4 \mathrm{sccm}$ the free molecular flow does not hold. This is in agreement with the results reported in Ref. [6], where at $1.2 \mathrm{sccm}$ the measured pattern strongly deviates from the free molecular flow simulation. Such a flow represents a Knudsen number of about $K_{n}=0.4$ according to Fig. 5, which is in the transition regime. 


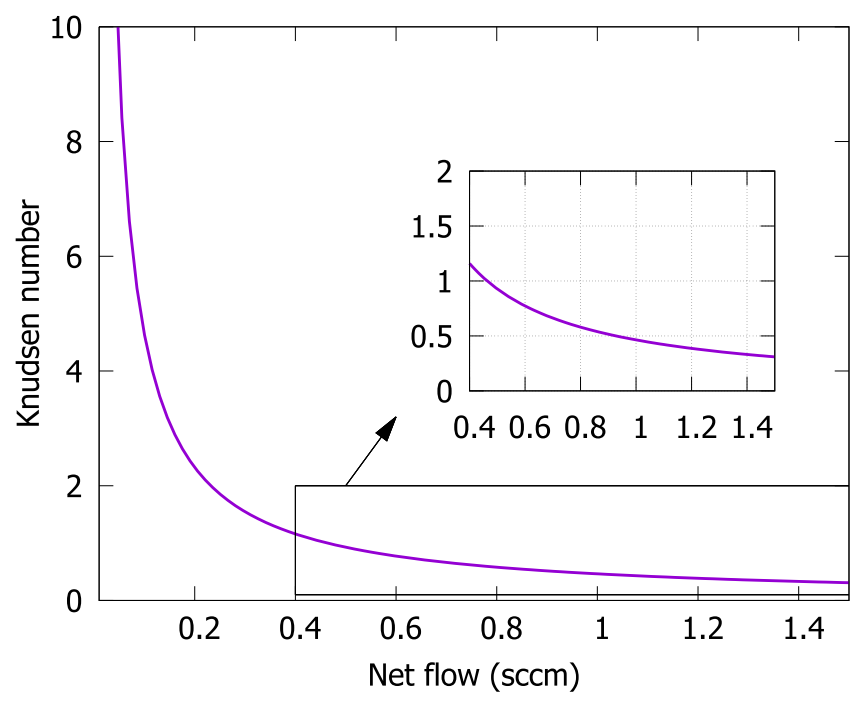

FIG. 5. Knudsen number in function of the net flow in the conical segment.

\section{CONCLUSION}

Solving the transport equation for the free molecular flow in complex systems with a Monte Carlo method requires sampling techniques to accelerate the convergence of the calculations. It was shown that path reversal can be used when it comes to cosine emitting surfaces, and that the transport equation could be solved using this method. Thus reverse path tracing is a sampling method that is easy to implement and that removes the need for surface discretization, improving both precision and accuracy. An example of these gains was illustrated with the computation of the flux ratio at the entrance of a pipe of aspect ratio $L / R=10$. Using the precision and accuracy gain of reverse path tracing, a density map was computed inside a conical section. The entrance density was then used to compute a net flow for which the free molecular flow regime would break down thus altering the performance of a controlled gas injector. Other local physical quantities, like pressure gradient, could have been computed using reverse path tracing but were left out since the goal of this paper was only to demonstrate the usefulness of this technique for vacuum system applications.

\section{ACKNOWLEDGMENTS}

We express our gratitude for the financial support from the Conseil de recherches en sciences naturelles et en génie du Canada (CRSNG) and the Fond de recherche Québécois sur la nature et les technologies (FRQNT).

[1] J. A. Fleming, VI. On a phenomenon of molecular radiation in incandescence lamps, London Edinburgh Dublin Philos. Mag. J. Sci. 16, 48 (1883).

[2] M. Knudsen, The laws of molecular flow and of inner friction flow of gases through tubes, J. Membr. Sci. 100, 23 (1909), The Early History of Membrane Science Selected Papers Celebrating vol. 100, Translated Version of 1995.

[3] P. Clausing, The flow of highly rarefied gases through tubes of arbitrary length, J. Vac. Sci. Technol. 8, 636 (1971), translated from German [Ann. Phys. (5) 12, 961 (1932)]. 
[4] R. P. Iczkowski, J. L. Margrave, and S. M. Robinson, Effusion of gases through conical orifices, J. Phys. Chem. 67, 229 (1963).

[5] J. A. Curless, Molecular beam epitaxy beam flux modeling, J. Vac. Sci. Technol. B 3, 531 (1985).

[6] L. Isnard and R. Ares, Theoretical and experimental molecular beam angular distribution studies for gas injection in ultra-high vacuum, J. Cryst. Growth 311, 1640 (2009).

[7] T. S. Cale and G. B. Raupp, A unified line of sight model of deposition in rectangular trenches, J. Vac. Sci. Technol. B 8, 1242 (1990).

[8] P. J. Lobo, F. Becheri, and J. Gomez-Goni, Comparison between monte carlo and analytical calculation of Clausing functions of cylindrical and conical tubes, Vacuum 76, 83 (2004).

[9] L. L. Carter and E. D. Cashwell, Particle Transport Simulation with the Monte Carlo Method, ERDA Critical Review Series (Technical Information Center, Oak Ridge, 1975).

[10] I. Lux and L. Koblinger, Monte Carlo Particle Transport Methods: Neutron and Photon Calculations (CRC Press, Boca Raton, FL, 1991).

[11] J. T. Kajiya, The rendering equation, SIGGRAPH Comput. Graph. 20, 143 (1986).

[12] J. R. Howell, M. P. Menguc, and R. Siegel, Thermal Radiation Heat Transfer, 6th ed. (CRC Press, Boca Raton, FL, 2015).

[13] C. P. Malhotra, R. L. Mahajan, and W. S. Sampath, High Knudsen number physical vapor deposition: Predicting deposition rates and uniformity, J. Heat Transfer 129, 1546 (2007).

[14] G. A Bird, Molecular Gas Dynamics and the Direct Simulation of Gas Flows, Oxford Engineering Science Series (Oxford University Press, New York, 1994).

[15] F. Sharipov and V. Seleznev, Data on internal rarefied gas flows, J. Phys. Chem. Ref. Data 27, 657 (1998).

[16] Y. Li, X. Chen, X. Bai, Q. Che, and Y. Li, A modified numerical method for the accurate calculation of molecular flow transmission probabilities and density distributions of cylindrical tubes, Vacuum 97, 60 (2013).

[17] C. M. Davies and C. B. Lucas, The failure of theory to predict the density distribution of gas flowing through a tube under free molecular conditions, J. Phys. D: Appl. Phys. 16, 1 (1983).

[18] F. Sharipov and Y. B. Barreto, Influence of gas-surface interaction on gaseous transmission probability through conical and spherical ducts, Vacuum 121, 22 (2015).

[19] R. Courant and D. Hilbert, Methods of Mathematical Physics, Wiley Classics Library (Wiley Interscience, New York, 1966).

[20] G. B. Arfken and H. J. Weber, Mathematical Methods for Physicists International Student Edition (Elsevier Science, San Diego, 2005).

[21] E M. Sparrow and A. Haji-Sheikh, Velocity profile and other local quantities in free-molecule tube flow, Phys. Fluids 7, 1256 (1964).

[22] J. O. Hirschfelder, C. F. Curtiss, and R. B. Bird, Molecular Theory of Gases and Liquids, Structure of Matter Series (John Wiley \& Sons, New York, 1964 [1954]).

[23] J. F. O'Hanlon, A User's Guide to Vacuum Technology (John Wiley \& Sons, New York, 2003). 